BUHEP-00-10

ROM2F $/ 2000 / 21$

\title{
RI/MOM Renormalization Window and Goldstone Pole Contamination
}

\author{
L. Giusti ${ }^{a}$ and A. Vladikas ${ }^{b}$ \\ ${ }^{a}$ Dept. of Physics, Boston University, \\ 590 Commonwealth Avenue, Boston MA 02215, USA. \\ ${ }^{b}$ INFN, Sezione di Roma II, c/o Dip. di Fisica, Univ. di Roma "Tor Vergata", \\ Via della Ricerca Scientifica 1, I-00133 Roma, Italy.
}

\begin{abstract}
We perform a comparative study of the ratio of lattice (Wilson fermion) renormalization constants $Z_{P} / Z_{S}$, obtained non-perturbatively from the RI/MOM renormalization conditions and from Ward Identities of on- and off-shell Green's functions. The off-shell Ward Identity used in this work relies on correlation functions with non-degenerate quark masses. We find that, due to discretization effects, there is a 10\%-15\% discrepancy between the two Ward Identity determinations at current bare couplings ( $\beta$ values). The RI/MOM result is in the same range and has a similar systematic error of $10-15 \%$. Thus, contrary to a previous claim, the contamination of the RI/MOM result from the presence of a Goldstone pole at renormalization scales $\mu \sim a^{-1}$ is subdominant, compared to finite cutoff effects.
\end{abstract}




\section{Introduction}

The Non-Perturbative (NP) renormalization technique proposed in [1] has been successfully applied to compute renormalization constants of two-fermion [2, 3] and four-fermion [4] operators with Wilson fermions. The standard implementation consists in imposing the RI/MOM renormalization conditions on conveniently defined amputated projected Green functions computed between offshell quark states of operators of interest, evaluated numerically at fixed momentum and lattice spacing (fixed lattice coupling $\beta$ ). In order to control discretization effects, one should work at renormalization scales $\mu$ well below the UV cutoff (i.e. $\mu \ll \mathcal{O}\left(a^{-1}\right)$ ). Moreover, any contribution in the renormalization constants due to spontaneous chiral symmetry breaking terms in the Green's functions has to be avoided to ensure that the non-perturbatively renormalized operators satisfy the standard QCD axial Ward Identities (abbreviated as WIs), i.e. $\Lambda_{Q C D} \ll \mu$. Thus, the existence of a "renormalization window" $\Lambda_{Q C D} \ll \mu \ll \mathcal{O}\left(a^{-1}\right)$ is essential to the reliability of the RI/MOM scheme in practical simulations, as was pointed out in ref. [1]. We note in passing that, as discussed in refs. [5, 4], the lower bound can be relaxed by matching renormalization conditions between a coarse and a fine lattice in the spirit of ref. [6]).

This has not always been the case at current values of the lattice coupling. In off-shell correlation functions, at high enough momenta, the dominant contributions are perturbative. For example, the leading non perturbative contribution of the pseudoscalar correlation function can be easily obtained from the Operator Product Expansion (OPE) of the quark propagator, which has been worked out in refs. [7]. Using either the lattice [8] or the Sum Rules [9] determination of the chiral condensate and quark mass values typical of present-day simulations, we estimate that the non perturbative contribution to the pseudoscalar correlation function at $\mu \simeq 2 \mathrm{GeV}$ is at most $2 \%$. Therefore, the existence of a "renormalization window' has been checked in [2, 10] by comparing the $p^{2}$ dependence of the Green's functions to the logarithmic one predicted by continuum perturbation theory 1 . In this sense, at $\beta=6.0,6.2$ and 6.4 , the scalar density renormalization constant $Z_{S}$ is well behaved in a large $\mu$-range, whereas there is no clear evidence of such a satisfactory renormalization window for the RI/MOM renormalization constant of the pseudoscalar density $Z_{P}$ (see ref. [2] for details).

Recently an alternative interpretation of the observed discrepancy between the RI/MOM and perturbative $Z_{P}$ results has been proposed in ref. [11]. It is suggested that the $p^{2}$ dependence of the pseudoscalar correlation function is due to large contributions of the spontaneous chiral symmetry breaking terms, which persist at scales used in present day simulations (i.e. $\mu \sim a^{-1}$ ). In other words, the lower bound of the renormalization window is not adequately satisfied. In order to test this proposal, the authors of ref. [11] analyze the pseudoscalar correlation function, taking into account the leading OPE non-perturbative contribution and ignoring discretization effects. They find that "a stricking and unexpected feature of the lattice data is the very large size of the Goldstone boson contribution to the pseudoscalar vertex".

In the present work we critically examine the claim of ref. [11] by comparing the RI/MOM

\footnotetext{
${ }^{1}$ Note that continuum perturbation theory converges much better than the lattice one. Moreover, higher orders have been calculated in the former case, whereas only 1-loop results are available in lattice perturbation theory.
} 
determination of the ratio $Z_{P} / Z_{S}$ to those obtained from two WIs at $\beta=6.2,6.4$ (the quenched approximation and Wilson fermions are implied throughout). WI results are unaffected by Goldstone pole contamination but are subject to discretization effects. Thus any discrepancy between two WI results gives an estimate of $\mathcal{O}(a)$ effects, whereas an eventually big discrepancy between the WI results and the RI/MOM ones would signal a Goldstone pole contamination in $Z_{P}$. We find that individual WI results for $Z_{P} / Z_{S}$ are characterized by errors of about $5 \%$ but they are discrepant to each other at the level of $10-15 \%$ due to $\mathcal{O}(a)$ effects. The RI/MOM result obtained at $\mu \sim 2 \mathrm{GeV}$ is compatible with the WI ones but has a larger error of about $10-15 \%$. Thus, contrary to the claim of ref. [11], we conclude that the RI/MOM determination of $Z_{P} / Z_{S}$ (and subsequently of $Z_{P}$ ) is affected by large discretization errors while any contamination from Goldstone pole contributions cannot be discerned. Analogous conclusions, based on an analysis of the form factors of the improved quark propagator, have been drawn in ref. [12].

Finally we wish to stress that the WI proposed in this paper (based on off-shell correlation functions and non-degenerate quark masses) has given a very stable estimate of $Z_{P} / Z_{S}$. In view of this, we prefer to evaluate $Z_{P}$ from the WI result of $Z_{P} / Z_{S}$ and the RI/MOM value of $Z_{S}$.

\section{The RI/MOM renormalization scheme and WIs}

In this section we review the basic characteristics of the RI/MOM renormalization scheme, implemented with the lattice regularization [1]. After giving the essential definitions, we discuss the compatibility of the RI/MOM scheme with (lattice) WIs at large renormalization scales $\mu$, where the presence of the Goldstone pole becomes negligible.

We start with basic definitions. Given the quark propagator $\mathcal{S}\left(x_{1}-x_{2} ; m\right)=\left\langle\psi\left(x_{1}\right) \bar{\psi}\left(x_{2}\right)\right\rangle$ and its Fourier transform $\mathcal{S}(p ; m)=\int d x \exp (-i p x) \mathcal{S}(x, m)$ (here $m$ is the quark mass), we define two "projections" (i.e. traces in spin and color space) as

$$
\begin{aligned}
& \Gamma_{\Sigma}(p ; m)=\frac{-i}{48} \operatorname{Tr}\left[\gamma_{\mu} \frac{\partial \mathcal{S}^{-1}(p ; m)}{\partial p_{\mu}}\right] \\
& \Gamma_{m}(p ; m)=\frac{1}{12} \operatorname{Tr}\left[\frac{\partial \mathcal{S}^{-1}(p ; m)}{\partial m}\right]
\end{aligned}
$$

We also consider bilinear quark operators of the form $O_{\Gamma}(x)=\bar{\psi}_{1}(x) \Gamma \psi_{2}(x)$ where $\psi_{f}(x)$ is the quark field and $\Gamma$ a generic Dirac matrix. For definitiveness we work with two different flavors $f=1,2$ with corresponding masses $m_{1}$ and $m_{2}$. Specific non-singlet bilinear operators will be denoted as $S(x), P(x)$ (scalar and pseudoscalar densities) and $V_{\mu}(x), A_{\mu}(x)$ (vector and axial currents). Given the insertion of the operator $O_{\Gamma}(x=0)$ in the 2-point fermionic Green's function

$$
G_{\Gamma}(p)=\int d x_{1} d x_{2} \exp \left[-i p\left(x_{1}-x_{2}\right)\right]\left\langle\psi\left(x_{1}\right) O_{\Gamma}(0) \bar{\psi}\left(x_{2}\right)\right\rangle
$$

and the corresponding amputated correlation function $\Lambda_{\Gamma}(p)=\mathcal{S}^{-1}(p) G_{\Gamma}(p) \mathcal{S}^{-1}(p)$, the projected 
amputated Green's function $\Gamma_{\Gamma}(p)$ is defined as

$$
\Gamma_{\Gamma}(p)=\frac{1}{12} \operatorname{Tr}\left[\mathrm{P}_{\Gamma} \Lambda_{\Gamma}(p)\right]
$$

where the trace is over spin and color indices and $\mathrm{P}_{\Gamma}$ is the Dirac matrix which renders the tree-level value of $\Gamma_{\Gamma}(p)$ equal to unity (i.e. it projects out the nominal Dirac structure of the Green function $\left.\Lambda_{\Gamma}(p)\right)$ :

$$
\begin{aligned}
& \mathrm{P}_{S}=I \quad ; \quad \mathrm{P}_{P}=\gamma_{5} \\
& \mathrm{P}_{V}=\frac{1}{4} \gamma_{\mu} \quad ; \quad \mathrm{P}_{A}=\frac{1}{4} \gamma_{5} \gamma_{\mu}
\end{aligned}
$$

Everything defined so far is a bare quantity, assumed to be regularized on the lattice 7 We opt for the lattice regularization scheme with Wilson fermions. Then the corresponding renormalized quantities in a given mass independent renormalization scheme are formally given by

$$
\begin{aligned}
\hat{m}_{f}(\mu) & =\lim _{a \rightarrow 0}\left[Z_{m}(a \mu) m_{f}(a)\right]=\lim _{a \rightarrow 0}\left[Z_{m}\left(m_{0 f}-m_{C}\right)\right] \\
\hat{\mathcal{S}}\left(p ; \hat{m}_{f}, \mu\right) & =\lim _{a \rightarrow 0}\left[Z_{\psi}(a \mu) \mathcal{S}\left(p ; m_{f}, a\right)\right] \\
\hat{\Gamma}_{\Sigma}\left(p^{2} / \mu^{2} ; m_{f}^{2} / \mu^{2}\right) & =\lim _{a \rightarrow 0}\left[Z_{\psi}^{-1}(a \mu) \Gamma_{\Sigma}\left(a p, a m_{f}\right)\right] \\
\hat{\Gamma}_{m}\left(p^{2} / \mu^{2} ; \hat{m}_{f}^{2} / \mu^{2}\right) & =\lim _{a \rightarrow 0}\left[Z_{\psi}^{-1}(a \mu) Z_{m}^{-1}(a \mu) \Gamma_{m}\left(a p ; a m_{f}\right)\right] \\
\hat{\Gamma}_{\Gamma}\left(p^{2} / \mu^{2} ; \hat{m}_{f}^{2} / \mu^{2}\right) & =\lim _{a \rightarrow 0}\left[Z_{\psi}^{-1}(a \mu) Z_{\Gamma}(a \mu) \Gamma_{\Gamma}\left(a p ; a m_{f}\right)\right]
\end{aligned}
$$

Note that $m_{0 f}$ denotes the bare quark mass of a given flavor, $m_{f}=m_{0 f}-m_{C}$ is power subtracted and logarithmically divergent and $\hat{m}_{f}$ is renormalized. Moreover, $Z_{\psi}^{1 / 2}$ is the quark field renormalization and $Z_{\Gamma}$ the operator renormalization. The functional dependence of the above expressions is determined by dimensional arguments and Lorenz invariance.

The renormalization constants in the RI/MOM scheme are defined by imposing the following off-shell conditions in the deep Euclidean region: the wave function renormalization $Z_{\psi}$ is obtained from

$$
\left.\hat{\Gamma}_{\Sigma}\left(p^{2} / \mu^{2} ; \hat{m}^{2} / p^{2}\right)\right|_{\substack{p^{2}=\mu^{2} \\ \hat{m}^{2}=0}}=\lim _{a \rightarrow 0} \lim _{m \rightarrow 0} Z_{\psi}^{-1}(a \mu) \Gamma_{\Sigma}\left(a \mu ; a^{2} m^{2}\right)=1
$$

the quark mass renormalization $Z_{m}$ is achieved through

$$
\hat{\Gamma}_{m}\left(p^{2} / \mu^{2} ; \hat{m}^{2} / p^{2}\right) \mid \begin{gathered}
p^{2}=\mu^{2} \\
\hat{m}^{2}=0
\end{gathered}=\lim _{a \rightarrow 0} \lim _{m \rightarrow 0} Z_{\psi}^{-1}(a \mu) Z_{m}^{-1}(a \mu) \Gamma_{m}\left(a \mu ; a^{2} m^{2}\right)=1
$$

\footnotetext{
${ }^{2}$ This means that the integrals of eq. (2) are really sums $\left(a^{8} \sum_{x_{1}, x_{2}}\right)$ which run over all lattice sites, labelled by $x_{1}, x_{2}$, etc. Also note that the $\mu$-dependence of renormalized correlation functions is sometimes suppressed; i.e. we use $\hat{\Gamma}(p ; \hat{m})$ instead of $\hat{\Gamma}\left(p^{2} / \mu^{2} ; \hat{m}^{2} / \mu^{2}\right)$.
} 
and for the bilinear operator $Z_{\Gamma}$ is obtained by imposing the renormalization condition

$$
\hat{\Gamma}_{\Gamma}\left(p^{2} / \mu^{2} ; \hat{m}^{2} / p^{2}\right) \mid \begin{aligned}
& p^{2}=\mu^{2} \\
& \hat{m}^{2}=0
\end{aligned}=\lim _{a \rightarrow 0} \lim _{m \rightarrow 0} Z_{\psi}^{-1}(a \mu) Z_{\Gamma}(a \mu) \Gamma_{\Gamma}\left(a \mu ; a^{2} m^{2}\right)=1
$$

For any fixed scale $\mu$, this procedure removes the UV divergences from all Green functions and thus renormalization is achieved. However, renormalization conditions must also be chosen so that the resulting renormalized operators transform "correctly" under the chiral group; i.e. they should belong to the same chiral representation as the nominal bare operators. This additional requirement, which ensures that chiral symmetry survives renormalization, is only true at large enough scales $\mu$. At small scales symmetry violating effects due to spontaneous chiral symmetry breaking in QCD should appear as $\Lambda_{Q C D}$-dependent form factors in the renormalized Green functions $\hat{\Gamma}_{\Gamma}\left(p^{2} / \mu^{2} ; \hat{m}^{2} / p^{2} ; \Lambda_{Q C D} / p^{2}\right)$. Moreover, in practical simulations, usually performed at (light) nonzero quark mass, explicit chiral symmetry breaking form factors, proportional to $\hat{m}(\mu)$ will also be present. Both types of form factors become negligible if the scale $\mu$ is adequately large. Therefore, the requirement $\Lambda_{Q C D} \ll \mu$ has to be satisfied in principle, so that quantities renormalized in the RI/MOM scheme respect chiral symmetry. Moreover, since one is usually working at fixed UV cutoff in simulations, the requirement $\mu \ll \mathcal{O}\left(a^{-1}\right)$ must be satisfied in order to control discretization errors. Thus, the reliability of the RI/MOM scheme on the lattice is ensured provided one is working in a renormalization window $\Lambda_{Q C D} \ll \mu \ll \mathcal{O}\left(a^{-1}\right)$.

The above general statements have been shown to be true in ref. [1] (and generalized for some cases of additive renormalization of dimension-six four-fermion operators in refs. [13, 4]). In particular it has been shown that the RI/MOM scheme is always compatible with vector WIs, whereas it is only compatible with the axial WIs at large scales $\mu$. The key observation is that the vector WIs

$$
\begin{aligned}
& \hat{\Gamma}_{S}(p ; \hat{m})=\hat{\Gamma}_{m}(p ; \hat{m}) \\
& \hat{\Gamma}_{V}(p ; \hat{m})=\hat{\Gamma}_{\Sigma}(p ; \hat{m})
\end{aligned}
$$

at momentum $p^{2}=\mu^{2}$, are automatically satisfied by the renormalized quantities $\hat{\Gamma}_{\Sigma}, \hat{\Gamma}_{m}, \hat{\Gamma}_{V}$ and $\hat{\Gamma}_{S}$ determined in the RI/MOM scheme by eqs. (6), (7) and (8). Conversely, if we use the RI/MOM scheme to fix, say, $\hat{\Gamma}_{\Sigma}$ and $\hat{\Gamma}_{m}$ (i.e. $Z_{\psi}$ and $Z_{m}$ ), then $Z_{V}$ and $Z_{S}$ (through the identity $Z_{S}=Z_{m}^{-1}$ ), as fixed by the WIs (9), are compatible to those obtained from the RI/MOM condition (8).

The compatibility of axial WIs to RI/MOM is more intricate; the proof would proceed in exactly the same way as in the vector case, if it were not for the extra terms on the l.h.s. of WIs

$$
\begin{aligned}
\hat{\Gamma}_{P}(p ; \hat{m})+\hat{m} \frac{\partial \hat{\Gamma}_{P}(p ; \hat{m})}{\partial \hat{m}} & =\hat{\Gamma}_{m}(p ; \hat{m}) \\
\hat{\Gamma}_{A}(p ; \hat{m}=0)+\lim _{q_{\rho} \rightarrow 0} \frac{q_{\mu}}{48} \operatorname{Tr}\left[\gamma_{5} \gamma_{\rho} \frac{\partial \hat{\Lambda}_{A}^{\mu}(p+q / 2, p-q / 2 ; \hat{m}=0)}{\partial q_{\rho}}\right] & =\hat{\Gamma}_{\Sigma}(p ; \hat{m}=0)
\end{aligned}
$$

which is the axial analogue to the vector WIs (9). Note that the second term of the l.h.s. of WIs (10) does not vanish in the chiral limit, due to the presence of a Goldstone pole. As shown in 
ref. [1] these extra terms are negligible in the deep Euclidean region $\Lambda_{Q C D} \ll p$. In this limit, the WIs and the RI/MOM conditions are compatible, just like the vector case. This limit corresponds to the lower bound of the renormalization window requirement, inherent in the RI/MOM scheme. Clearly, all statements made thus far are true up to discretization effects, which are present both in the RI/MOM and the WI implementations on the lattice. We will address this problem at a later stage of this work. What we need to consider at present, is that RI/MOM results are reliable in the renormalization window $\Lambda_{Q C D} \ll p \ll \mathcal{O}\left(a^{-1}\right)$, whereas WI results are reliable in the window $p \ll \mathcal{O}\left(a^{-1}\right)$.

The main aim of the present work is to establish whether there is significant Goldstone pole contamination of the lattice RI/MOM results obtained thus far. In particular, the RI/MOM determination of $Z_{P} / Z_{S}$ at current $\beta$ values and momenta does not display a clear plateau; see refs. [1]-[3]. The problem may be either due to the Goldstone pole problem or, as claimed in ref. [12], due to discretization effects 5 . Since the WI determination of the ratio $Z_{P} / Z_{S}$ does not suffer from Goldstone pole contamination, comparison of the WI and the RI/MOM results for this quantity should offer a way of estimating this effect. For example, the WI result from eqs. (9) and (10) is

$$
\frac{Z_{P}}{Z_{S}}=\frac{\frac{\Gamma_{S}}{\Gamma_{P}}}{1+\frac{m}{\Gamma_{P}} \frac{\partial \Gamma_{P}}{\partial m}}
$$

whereas the RI/MOM result from eq. (8) is

$$
\frac{Z_{P}}{Z_{S}}=\frac{\Gamma_{S}}{\Gamma_{P}}
$$

The denominator on the r.h.s. of the WI (11) is missed by the RI/MOM eq. (12) and this results to a Goldstone pole contamination of the latter. This particular WI determination, however, is difficult to implement in practice (e.g. realization of derivatives w.r.t. the quark mass in lattice numerical simulations etc.). In the next section we discuss several WIs, which are equivalent (up to discretization effects) but more useful in practice.

\section{The ratio $Z_{P} / Z_{S}$ from WIs}

We will now review three methods, based on WIs, for the determination of the scale independent ratio $Z_{P} / Z_{S}$. A first method consists in computing $Z_{P} / Z_{S}$ as the ratio of the PCAC current quark mass to the bare (subtracted) quark mass [6]. In standard notation (see refs. [15], [16]) the current quark mass for Wilson fermions is obtained from the standard PCAC relation (an axial WI, valid $\forall x \neq 0)$ :

$$
2\left[m_{0}-\bar{m}\left(m_{0}\right)\right]=Z_{A} \frac{\partial_{0} \int d^{3} x\left\langle A_{0}(x) P(0)\right\rangle}{\int d^{3} x\langle P(x) P(0)\rangle}
$$

\footnotetext{
${ }^{3}$ Moreover, the 1-loop Perturbation Theory PT (and Boosted PT -BPT) result for $Z_{P}$ is in stark disagreement with the RI/MOM estimate. This comparison, however, does not reveal much, as the BPT result also suffers from $\mathcal{O}\left(g_{0}^{4}\right)$ errors.
} 
whereas the mass power subtraction $m_{C}$ can be obtained by linearly extrapolating either the square of the pion mass $m_{\pi}^{2}$ or $\left[m_{0}-\bar{m}\left(m_{0}\right)\right]$ to their vanishing value. Then vector and axial WIs (see refs. 17, 15, 16]) determine the quark mass renormalization

$$
\hat{m}=Z_{S}^{-1}\left[m_{0}-m_{C}\right]=Z_{P}^{-1}\left[m_{0}-\bar{m}\right]
$$

and thus by computing $\left[m_{0}-\bar{m}\right]$ at several $m_{0}$ 's the ratio $Z_{P} / Z_{S}$ is obtained as the slope of

$$
m_{0}-\bar{m}=\frac{Z_{P}}{Z_{S}}\left[m_{0}-m_{C}\right]
$$

This determination does not depend on the critical quark mass $m_{C}$. It does, however, depend on the determination of $Z_{A}$. Since the methods relies on the WI (13) on hadronic states, we will label it WIh.

A second method consists in combining eq. (15) with the axial WI

$$
\left(m_{01}+m_{02}-2 \bar{m}\right) \Gamma_{P}\left(a p ; a m_{1}, a m_{2}\right)=\frac{1}{12} \operatorname{Tr} \mathcal{S}^{-1}\left(a p ; a m_{1}\right)+\frac{1}{12} \operatorname{Tr} \mathcal{S}^{-1}\left(a p ; a m_{2}\right)
$$

(considered in the mass-degenerate case), in order to obtain

$$
\frac{Z_{P}}{Z_{S}}=\frac{\operatorname{Tr} \mathcal{S}^{-1}(a p ; a m)}{12\left[m_{0}-m_{C}\right] \Gamma_{P}(a p ; a m, a m)}
$$

This determination requires the projected amputated correlation functions and the quark propagators in momentum space. We anticipate at this stage, that this method fails in practice. This is due to the large discretization errors of the quark propagator (a detailed investigation of these effects, which remain large even when Clover improvement is implemented beyond tree-level, has been performed in ref. [12]).

A third method consists in combining eq. (16) for the degenerate mass case with the vector WI

$$
\left(m_{02}-m_{01}\right) \Gamma_{S}\left(a p ; a m_{1}, a m_{2}\right)=-\frac{1}{12} \operatorname{Tr} \mathcal{S}^{-1}\left(a p ; a m_{1}\right)+\frac{1}{12} \operatorname{Tr} \mathcal{S}^{-1}\left(a p ; a m_{2}\right)
$$

so as to eliminate the quark propagators. Using also eq. (15) we obtain

$$
\frac{Z_{P}}{Z_{S}}=\frac{\left(m_{1}-m_{2}\right) \Gamma_{S}\left(a p ; a m_{1}, a m_{2}\right)}{m_{1} \Gamma_{P}\left(a p ; a m_{1}, a m_{1}\right)-m_{2} \Gamma_{P}\left(a p ; a m_{2}, a m_{2}\right)}
$$

The advantage of this determination is that the large $\mathcal{O}(a p)$ discretization errors, present in the quark propagator (cf. ref. [12]), are eliminated. Since this determination depends on correlation functions of external quark states, we will label it WIq. Note that this method is characterized by non-degenerate quark masses.

Finally, we mention that a WI with hadronic states has also been used for the computation of the ratio $Z_{P} / Z_{S}$; see refs. [16, 18]. 


\section{Results}

Our results are based on earlier simulations; all technical details can be found in refs. [2, 14]. Here we just mention that the dataset we analyze has been obtained with the Wilson (unimproved) action, at couplings $\beta=6.2$ and 6.4 and at several hopping parameters in the range of the strange quark mass. All results shown here are extrapolated to the chiral limit. The RI/MOM results are those of ref. [2] (which agree with the ones of ref. [3]) whereas the WIh and WIq results are new. We mostly show the Wilson $\beta=6.2$ case 1 .

In figure 1 we show the data and linear fit characteristic of the method WIh. The slope of the fitting line is $Z_{P} /\left[Z_{S} Z_{A}\right]$. In order to extract $Z_{P} / Z_{S}$ from the slope, we use the RI/MOM estimate for $Z_{A}$, as given in ref. [2]. This is in accordance (with larger errors) to the RI/MOM result of ref. $[3]$.

Figure 1: The value of $2\left[m_{0}-\bar{m}\right] / Z_{A}$, obtained from eq. (13), as a function of the Wilson hopping parameter $1 / K$ at $\beta=6.2$. The slope of the linear fit is the WIh result for $Z_{P} /\left[Z_{S} Z_{A}\right]$, according to eq. (15). The $K_{C}$ value implicit in the abscissa axis is determined from the vanishing of $m_{\pi}^{2}$ (linear in $1 / K)$.

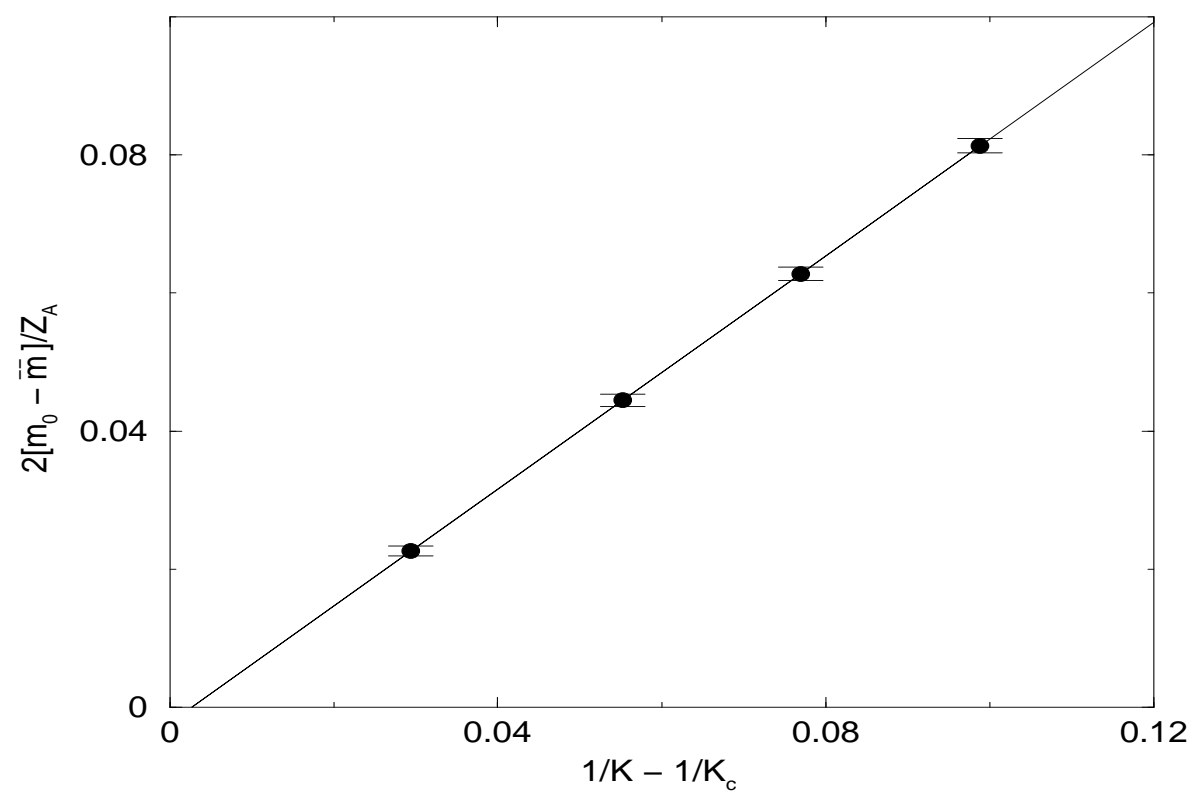

In Fig. 2(a) we show our $\beta=6.2$ results obtained from WI (17), as a function of the (lattice) scale

$$
a^{2} \bar{\mu}^{2}=\sum_{\nu} \sin ^{2}\left(a \mu_{\nu}\right)
$$

\footnotetext{
${ }^{4}$ Note that our $\beta=6.0$ dataset has also been analyzed, with qualitatively similar results.
} 
Due to enormous systematic errors, our results range over a large interval and no plateau is seen. The tree-level value of eq. (17) is given by

$$
\frac{Z_{P}}{Z_{S}}=1+\frac{2 \sum_{\nu} \sin ^{2}\left(a p_{\nu} / 2\right)}{a m}
$$

where the second term on the r.h.s. is the tree-level $\mathcal{O}(a)$ effect arising from the propagator sum in the numerator of eq. (17). In Fig. 2(b) we have corrected our results by this factor, in the spirit of KLM tree-level improvement (cf. ref. [19]). Although this factor accounts for a big part of the discretization errors, the remaining effects are still large and render the method unreliable.

Figure 2: $Z_{P} / Z_{S}$ results at $\beta=6.2$. (a) Results obtained from WI (17); (b) Results corrected by the KLM factor of eq. (21).

(a)

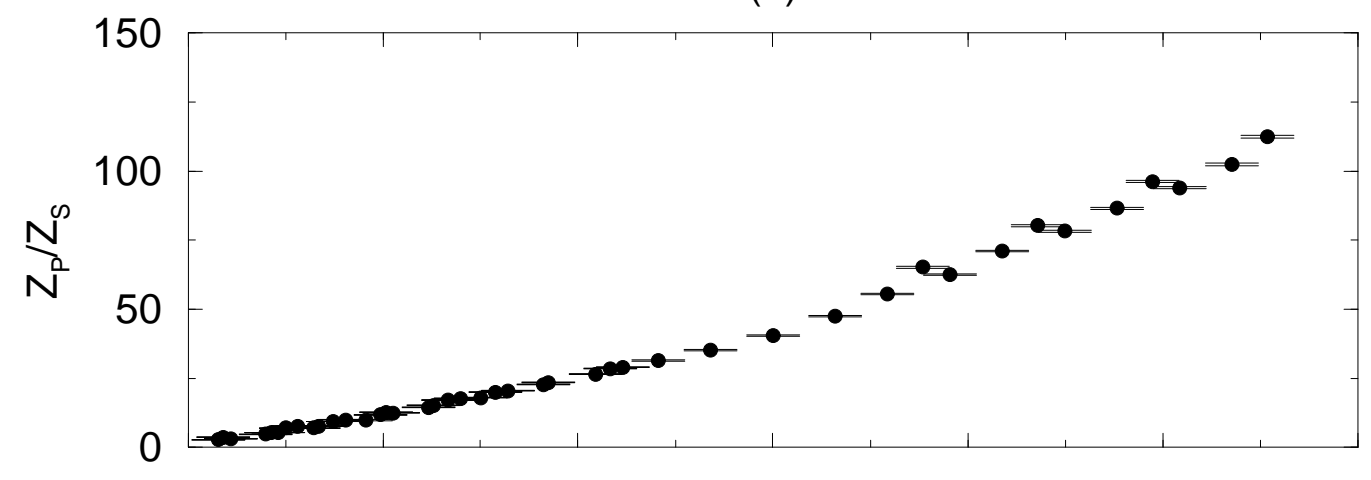

(b)

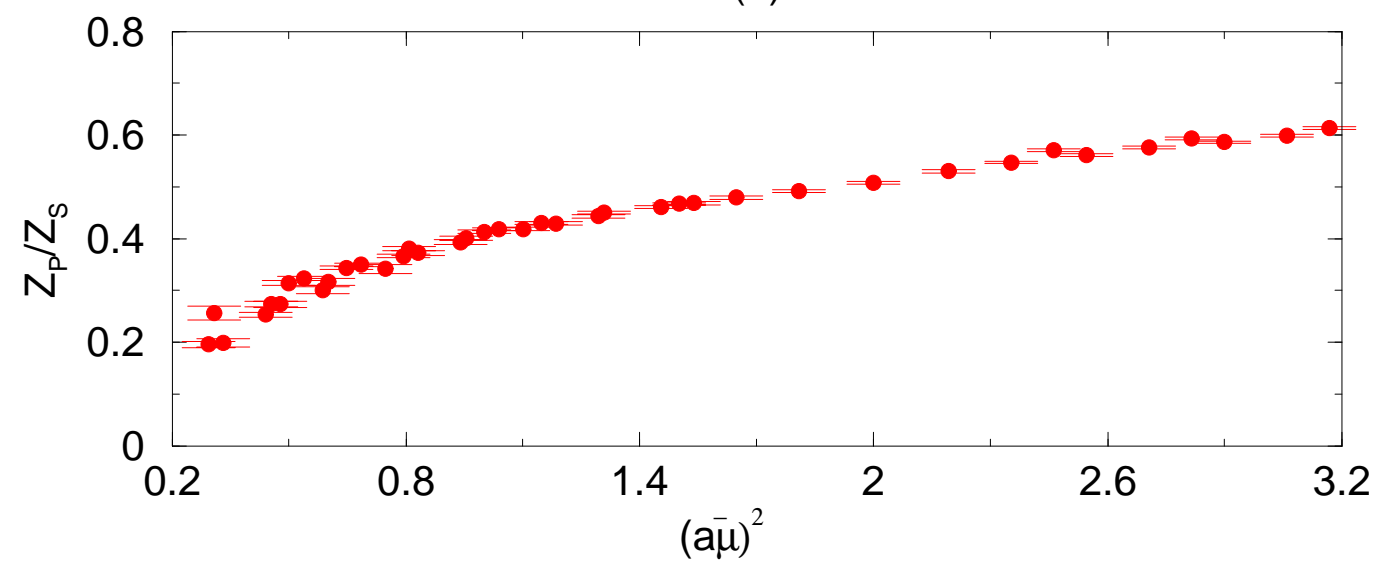

In Fig. 3 we compare the $\beta=6.2$ results for the RI/MOM and WIq determinations, as a function of the (lattice) scale $a^{2} \bar{\mu}^{2}$. It is clear that at small scales there is a large discrepancy 
due to the sizeable Goldstone pole contamination of the RI/MOM method, which decreases with increasing scale. At large scales it is impossible to discern whether the discrepancy is due to a remnant Goldstone pole contamination or the discretization effects. Moreover, even at larger scales, the renormalization window of the RI/MOM method, compared to the plateau displayed by the WIq results, is rather poor. In ref. [2], the central values of the RI/MOM results were taken at $(a \bar{\mu})^{2} \simeq 0.8$ and the systematic errors estimated as the spread of the values in the region $0.8 \leq(a \bar{\mu})^{2} \leq 1.5$. This choice takes into account the instability of the renormalization window, yielding a RI/MOM result with large errors. On the contrary, the WI data displays a good plateau already at rather small scales. Our choice $(a \bar{\mu})^{2} \in[0.2,0.8]$ for the WIq plateau gives very accurate results.

Figure 3: $Z_{P} / Z_{S}$ results at $\beta=6.2$. (a) WIq and $\mathrm{RI} / \mathrm{MOM}$ determinations as a function of the renormalization scale. (b) Enlargement of (a), highlighting the $\mu$ values where scale independence is expected.

(a)

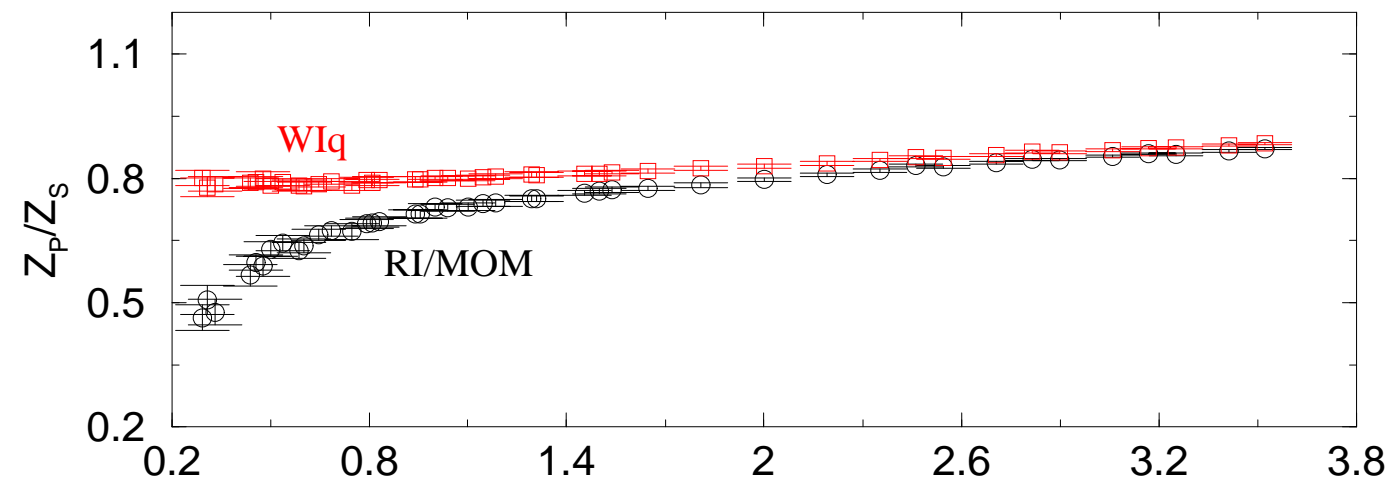

(b)

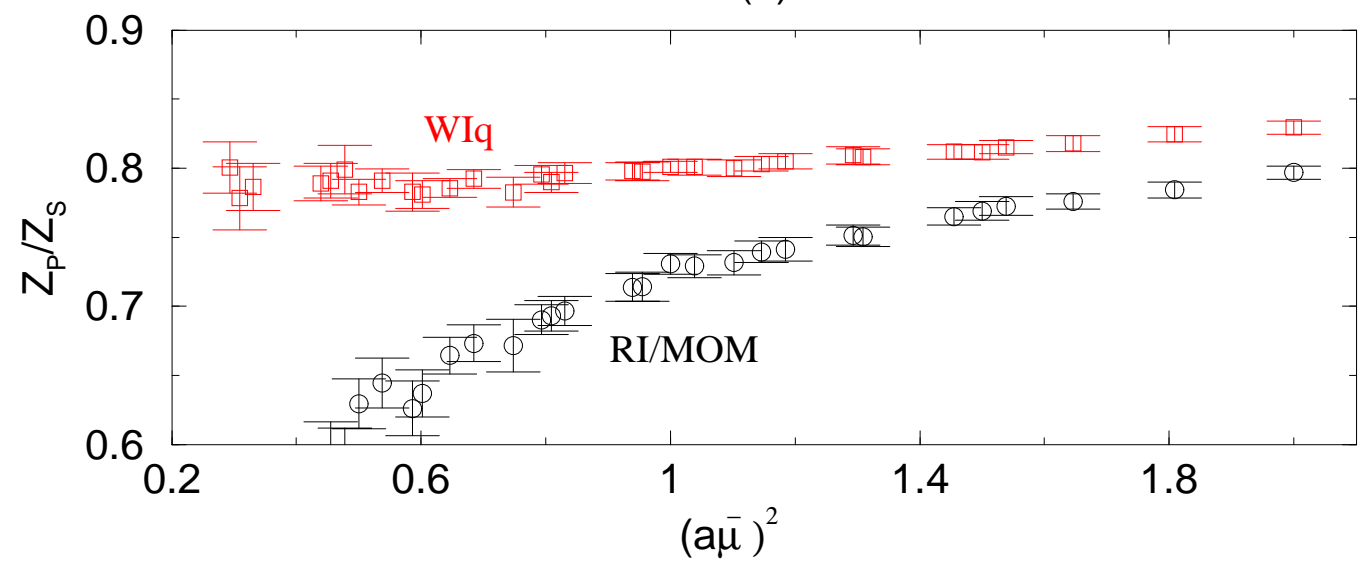

In order to estimate the discretization errors, we collect in Fig. 1 , the various determinations 
of $Z_{P} / Z_{S}$ at $\beta=6.2$ and 6.4. The most accurate estimates are those based on the two WIs (WIh and WIq). They are not, however fully compatible, due to discretization effects (recall that WI results do not suffer from the Goldstone pole problem). This indicates that these effects introduce a systematic error of $\simeq 15 \%$ at $\beta=6.2$, which decreases to $\simeq 10 \%$ at $\beta=6.4$. The RI/MOM determination, which is far less accurate due to the instability of the renormalization window, is nevertheless in the same range of values. Thus, it is clear that any remnant Goldstone pole contamination in the RI/MOM result is not superior in magnitude to the uncertainties arising from discretization effects. Also, the fact that the spread of the various determinations of $Z_{P} / Z_{S}$ decrease with increasing $\beta$, indicates that the discrepancies are due to (decreasing) finite cutoff effects.

Figure 4: $Z_{P} / Z_{S}$ results (a) at $\beta=6.2$; (b) at $\beta=6.4$. Comparison of non-perturbative (WIq, WIh, RI/MOM) and perturbative (BPT, PT) determinations.

(a)

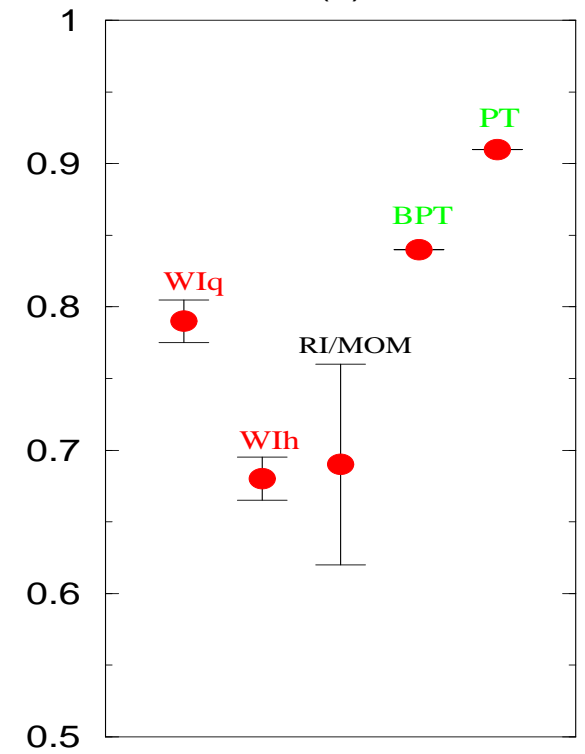

(b)

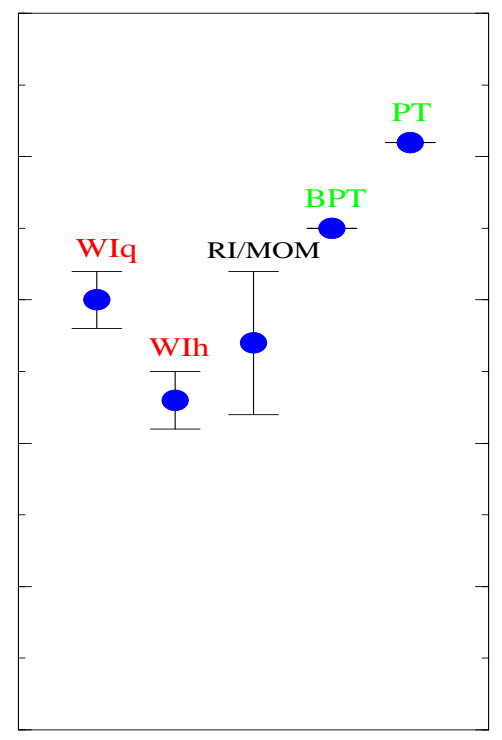

Our new WI results demonstrate that the discrepancy of $10-15 \%$ for $Z_{P} / Z_{S}$ carries over to $Z_{P}$, computed (non-perturbatively) from

$$
Z_{P}=\left[\frac{Z_{P}}{Z_{S}}\right]^{\mathrm{WI}} Z_{S}^{\mathrm{RI} / \mathrm{MOM}}
$$

In practice we use the WIq determination for the ratio $Z_{P} / Z_{S}$ in the above equation; i.e. combining eq. (19) with the RI/MOM renormalization condition (8) for the scalar density, we have

$$
Z_{P}=Z_{\psi} \frac{m_{1}-m_{2}}{m_{1} \Gamma_{P}\left(a \mu ; a m_{1}, a m_{1}\right)-m_{2} \Gamma_{P}\left(a \mu ; a m_{2}, a m_{2}\right)} .
$$




\begin{tabular}{||c|c|ccc||}
\hline \hline$\beta$ & Method & $Z_{P}$ & $Z_{S}$ & $Z_{P} / Z_{S}$ \\
\hline \hline \multirow{6}{*}{6.2} & PT & 0.78 & 0.86 & 0.91 \\
& BPT & 0.65 & 0.77 & 0.84 \\
& RI/MOM [2] & $0.50(5)$ & $0.72(1)$ & $0.69(7)$ \\
& WIq [This work] & $0.57(1)$ & - & $0.79(2)$ \\
& WIh [This work] & $0.50(2)$ & - & $0.68(2)$ \\
\hline \multirow{6}{*}{6.4} & PT & 0.79 & 0.86 & 0.91 \\
& BPT & 0.67 & 0.79 & 0.85 \\
& RI/MOM [2] & $0.57(4)$ & $0.74(1)$ & $0.77(5)$ \\
& WIq [This work] & $0.60(1)$ & - & $0.80(2)$ \\
& WIh [This work] & $0.54(2)$ & - & $0.73(2)$ \\
\hline \hline
\end{tabular}

Table 1: Perturbative (PT and BPT) and non-perturbative (RI/MOM, WIq, WIh) estimates of renormalization constants. The RI/MOM values are at $\bar{\mu}^{2} a^{2}=0.8$. The WIq and WIh results are obtained from eq. (22).

The wave-function renormalization constant $Z_{\psi}$ has been computed in ref. [2]. In Table 1] we collect our results for $Z_{P}, Z_{S}$ and their ratio. Consequently, at these $\beta$ values, determinations of the quark mass (and chiral condensate) which differ in the choice of non-perturbative renormalization (i.e. WIq, WIh, RI/MOM), would also display the same variations f. These differences should vanish upon extrapolation to the continuum limit.

It is interesting to note that, upon expressing the first of WIs (18) in terms of renormalized quantities and using the RI/MOM renormalization condition for $\Gamma_{S}$, the relation

$$
\left[\hat{m}_{1}(\mu)-\hat{m}_{2}(\mu)\right]^{\mathrm{RI} / \mathrm{MOM}}=\left[\frac{1}{12} \operatorname{Tr} \hat{\mathcal{S}}^{-1}\left(\mu ; \hat{m}_{1}\right)-\frac{1}{12} \operatorname{Tr} \hat{\mathcal{S}}^{-1}\left(\mu ; \hat{m}_{2}\right)\right]^{\mathrm{RI} / \mathrm{MOM}}
$$

can readily be obtained. This gives a non-perturbative quark-mass determination which does not require knowledge of $Z_{S}$ (or $Z_{P}$ ); cf. ref. [12]. Moreover, it does not suffer from large $\mathcal{O}(a p)$ discretization effects (they cancel in the difference of quark propagators at any quark mass values), which would otherwise have to be isolated by chiral extrapolation. This determiantion does however require the computation of the wave function renormalization $Z_{\psi}$ in the RI/MOM scheme. Since this is a mass-independent renormalization scheme, the above can only be implemented in the limit $m^{2} / \mu^{2} \ll 1$.

\section{Acknowledgments}

We wish to thank V. Lubicz, G. Martinelli and S. Sint for numerous illuminating discussions. A.V. thanks the DESY Theory Group for its hospitality during the early stages of this work. L. G. has

\footnotetext{
${ }^{5}$ In refs. [14, 8], a good agreement was observed between the quark mass (and chiral condensate), renormalized with $Z_{S}$ and that renormalized with $Z_{P}$ in the RI/MOM scheme. This is equivalent to the good agreement between the central values of the WIh and RI/MOM estimates of $Z_{P} / Z_{S}$. It is clear from fig.
} 
been supported in part under DOE grant DE-FG02-91ER40676.

\section{References}

[1] G. Martinelli et al., Nucl. Phys. B445 (1995) 81.

[2] V. Gimenez, L. Giusti, F. Rapuano and M. Talevi, Nucl. Phys. B531 (1998) 429.

[3] M. Göckeler at al., Nucl. Phys. B544 (1999) 699.

[4] A. Donini et al., Eur. Phys. J. C10 (1999) 121.

[5] G. Martinelli et al., Phys. Lett. B411 (1997) 141.

[6] K. Jansen et al., Phys. Lett. B372 (1996) 275;

M. Lüscher et al., Nucl.Phys. B491 (1997) 323.

[7] H.D. Politzer, Nucl.Phys. B117 (1976) 397;

P. Pasqual and E. de Rafael, Z. Phys. C12 (1982) 127;

M.J. Lavelle and M. Oleszczuk, Phys. Lett. B275 (1992) 133.

[8] L. Giusti, F. Rapuano, M. Talevi and A. Vladikas, Nucl. Phys. B538 (1999) 249.

[9] S. Narison, Nucl. Phys. (Proc. Suppl.) 86 (2000) 242 and references therein.

[10] S. Capitani et al., Nucl. Phys. B544 (1999) 669.

[11] J-R. Cudell, A. Le Yaouanc and C. Pittori, Phys. Lett. B454 (1999) 105.

[12] D. Becirevic, V. Gimenez, V. Lubicz and G. Martinelli, Phys. Rev. D61 (2000) 114507.

[13] M. Testa, Nucl. Phys. B (Proc. Suppl.) 63 (1998) 38;

JHEP 04 (1998) 002.

[14] V. Gimenez, L. Giusti, F. Rapuano and M. Talevi, Nucl. Phys. B540 (1999) 472.

[15] M. Bochicchio et al., Nucl. Phys. B262 (1985) 331.

[16] M .Crisafulli, V. Lubicz and A. Vladikas, Eur. Phys. J. C4 (1998) 145.

[17] L.H. Karsten and J. Smit, Nucl. Phys. B183 (1981) 103.

[18] L. Maiani and G. Martinelli, Phys. Lett. B178 (1986) 265;

G.Martinelli, S. Petrarca, C.T. Sachrajda and A. Vladikas Phys. Lett. B311 (1993) 241;

Phys. Lett. B317 (1993) 660;

D.S. Henty et al. (UKQCD collaboration), Phys. Rev. D51 (1995) 5323.

[19] G.P. Lepage, Nucl. Phys. (Proc. Suppl.) 26 (1992) 45;

A.S. Kronfeld, Nucl. Phys. (Proc. Suppl.) 30 (1993) 445;

P.B. Mackenzie, Nucl. Phys. (Proc. Suppl.) 30 (1993) 35. 